\title{
FEASIBILITY OF THE CONCEPT OF ORALLY ADMINISTERED ELECTRONIC BOLUSES FOR IDENTIFICATION OF CATTLE
}

\author{
Kerstin E. Müller \\ Department of Farm Animal Health, Division Ruminant Health, Faculty of Veterinary Medicine, \\ Utrecht University, The Netherlands \\ Visiting address: Yalelaan 7, 3584 CL Utrecht. Postal address: PO Box 80.151, 3508 TD Utrecht, \\ The Netherlands
}

\begin{abstract}
The European Community Directive 92/102 requires all member countries to be able to operate a national identity scheme for cattle, sheep, pigs and goats with brief details kept in a central register. An identification device must be accurate, dependable and tamperproof. At the same time, it has to safeguard the welfare of the animal. The present study was performed to examine the effects of electronic boluses for animal identification on the health of neonatal calves.

Electronic boluses for animal identification (electronic bolus, NEDAP AGRI, Groenlo, the Netherlands) were administered into the ruminoreticular compartment of 5 neonatal calves aged from 3 to 5 days with a bodyweight from 36 to $42 \mathrm{~kg}$, which were purchased from a local dealer. An applicator was used which allowed insertion of the boluses directly into the reticulorumen. In order to examine possible effects of these boluses on the health of the calves, the animals were closely monitored during a period of 3 months by a) general clinical examination and b) analyses of various blood parameters at regular intervals. Five additional calves, which received no bolus, served as controls. Once weekly the following blood parameters were determined: PCV, RBC, WBC and differentials, serum total protein and protein pattern, urea, $\mathrm{Na}, \mathrm{K}$ and $\mathrm{Cl}$.

No diseases occurred that were directly related to the presence of the bolus in the gastrointestinal tract (e.g. esophageal obstruction, obstruction of the pylorus) during a period of 3 months. The number of episodes of diarrhea or bronchopneumonia in the group fitted with boluses did not differ from untreated controls. Results of laboratory analyses revealed no statistically significant differences in the group fitted with boluses compared to controls. Weight gains in calves with boluses did not differ from controls. X-rays taken at the end of the trial showed that all boluses were sited in the reticulum of the calves. When the boluses were read at an age of 12 months, all boluses were detected. In a second trial, 4 female calves born at the department itself received a bolus transponder at 3 days of age. These animals were monitored by clinical examination once weekly until an age of one year. These calves did not show any disease symptoms and no boluses were lost within one year.

Based on findings in 9 calves, which were closely monitored, there was no evidence for bolus associated effects on the general health. Under the precaution that no force is used the applicator specifically developed for intraruminal administration of boluses is suitable for use.
\end{abstract}

